\title{
THE EFFECTIVENESS OF WATER MIST IN THE FUNCTIONING OF AUTOMATIC FIRE EXTINGUISHING SYSTEMS
}

\author{
Galina S. Nyashina ${ }^{a}$ \\ ${ }^{1}$ National Research Tomsk Polytechnic University, 634050 Tomsk, Russia
}

\begin{abstract}
Currently, one of the most perspective directions fire protection is the use of water mist extinguishing agents. An experimental study of phase transformations of water mist droplets (radius of 50-500 microns) in motion in a high-temperature $(500-1800 \mathrm{~K})$, typical products of combustion of petroleum products (gasoline, kerosene, acetone, alcohol). Use high speed (the speed of recording at least 105 images per second) and optical methods of recording streams of liquid and gas medium. The influence of the parameters of the test process (the initial temperature and the initial droplet size) at the rate of evaporation of atomized water under these conditions. The optimum droplet size histograms in extinguishing fluid stream, providing evaporation of atomized water flow.
\end{abstract}

\section{Introduction}

Most of the objects oil and gas industry are highly explosive. One of the ways to prevent the development of emergency situations, is to equip the production facilities with modern fire protection systems.

Currently, a popular means of fire suppression built and reconstructed facilities were modern systems using water mist. Features such systems have made them almost ideal for fighting fires. The main advantage of extinguishing expansion water mist is a high fire extinguishing efficiency with minimal impact on their application for building and property thereon [1].

Water mist cools, shields heat radiation and reduces the oxygen concentration. Water boils when falls into the fire. Due to the very high specific heat of vaporization $-2.26 \mathrm{MJ} / \mathrm{kg}$ when boiling water is an effective heat removal from the combustion zone. This can cause to a complete cessation of the combustion reaction. Furthermore, upon evaporation of water in the high temperature region formed by vapor which prevents gas exchange at the time of the combustion products with the ambient air, and also reduces the oxygen concentration near the combustion zone. Thus, the water, in addition to the cooling mechanism implements two extinguishing insulation and dilution.

Modular expansion water mist completely autonomous. electricity and additional water containers are required. An important feature is the actual water mist harmless to humans. Therefore, in the near future, these systems need to be widely disseminated [2].

The purpose of this work is the experimental study of phase transformations of the dispersed water droplets as they move in a few high-temperature combustion products of the most common liquid petroleum products using advanced optical techniques of digital "tracer" visualization and high-speed video recording.

\section{Experimental method and results of research}

Cycle experiments included two series. In the first series of experiments recorded videos sprayed operating the water entering the cylindrical channels with flames. In the second series of recorded images of droplets after passing the flame of the combustion zone. Motional sprayed the working fluid in the high temperature gas environment recorded by high-speed cameras with an aspect ratio $-1024 \times 1024$ pixels and a frequency of more than 1000 images per second, as well as the cross-correlation cameras with an aspect ratio $-2048 \times 2048$ pixels, the minimum delay between two successive frames - no more 5 microseconds. Videos transferred to a workstation, which processes it using a software package «Tema Automotive». In the experiment with spraing water flows used flammable liquids such as gasoline, kerosene, acetone, industrial alcohol.

The studies were prepared depending. Figure 1a shows the dependence of the parameter characterizing the change of the sizes drops of water when moving through a high temperature gas environment $\Delta R$ from the initial temperature of the liquid. Change of the initial temperature $T_{\mathrm{w}}$ dispersed water droplets observed that increasing the initial temperature of the water $10 \mathrm{~K}$ even in the range to the ability to increase the intensity of the two droplet evaporation process.

\footnotetext{
${ }^{a}$ Corresponding author : gsn1@tpu.ru
} 


\section{MATEC Web of Conferences}
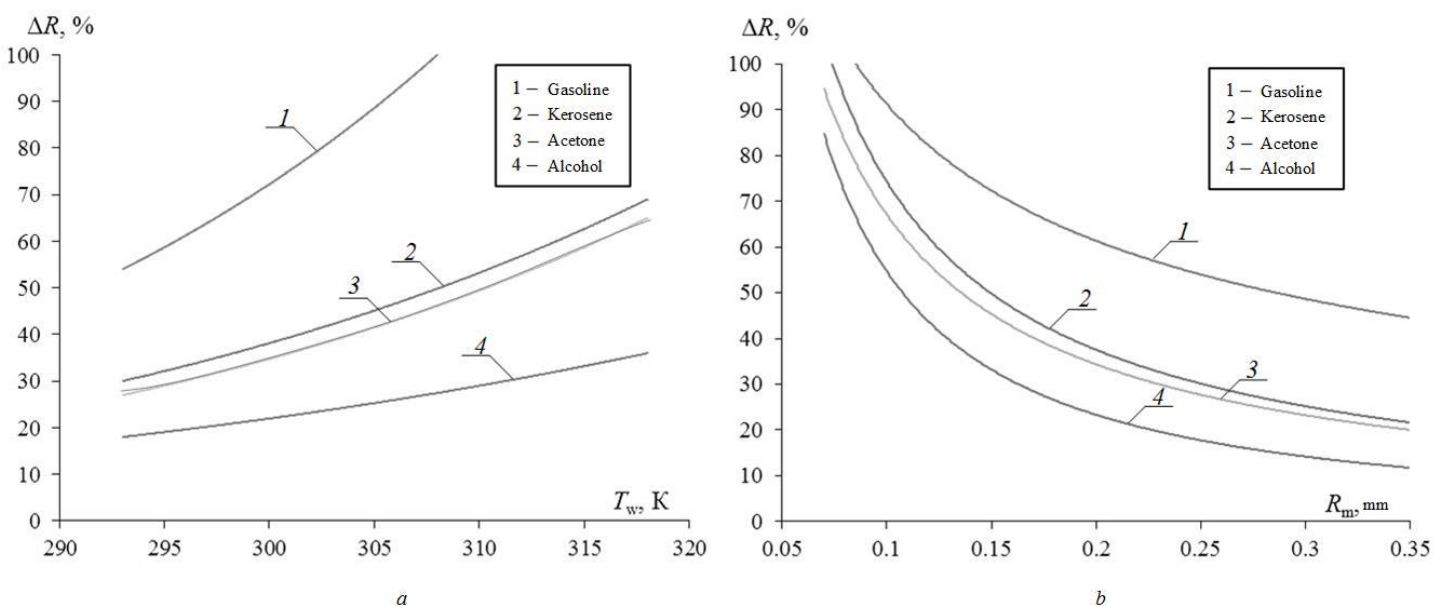

Figure 1. Experimental depending of the parameter $\Delta R$ from the initial droplet size $R_{\mathrm{m}}(a)$ and the initial fluid temperature $T_{\mathrm{w}}(b)$

Figure $1 b$ shows the parameter $\Delta R$ on the initial droplet size. In this case, it can be concluded that the water mist by reducing the evaporation time, increasing the heat transfer coefficient and absorption capacity with decreasing droplet diameter is more suitable for extinguishing gruboraspylennaya than water. However, there is a limit in the amount of drops. This conclusion was obtained in the pilot study process "braking" and ash drops high temperature gases.

Figure 2 shows the video recordings combined liquid droplets and the velocity field at the inlet and outlet of the flame.

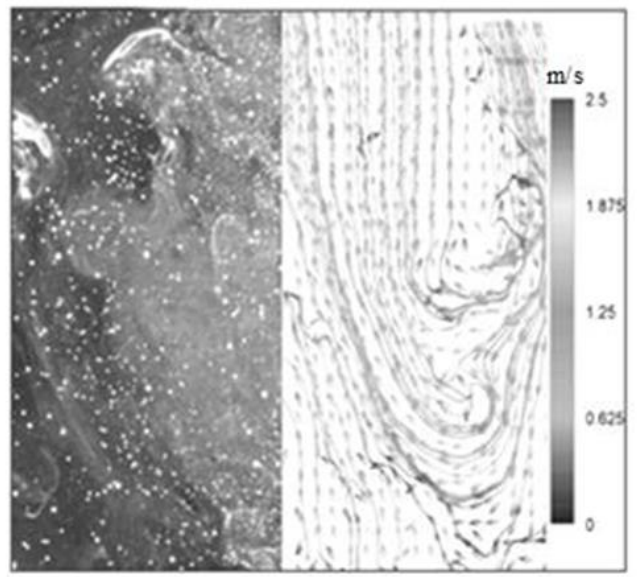

a

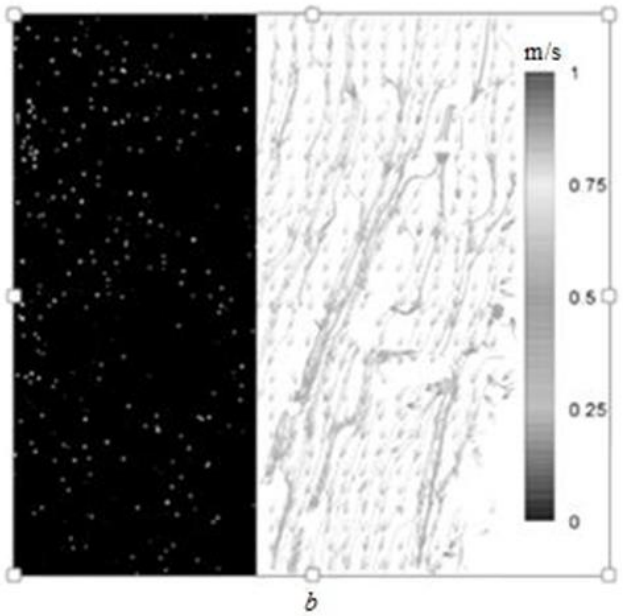

Figure 2. Videograms combined liquid droplet and the velocity field "tracer" particle inlet $(a)$ and the output $(b)$ of the flame at the initial gas velocity $U_{\mathrm{g}}=1 \mathrm{~m} / \mathrm{s}$ and droplets $U_{\mathrm{m}}=1 \mathrm{~m} / \mathrm{s}$

As shown in Figure 2 of the particles that hit the gas flow deviated from its trajectory and flew off to the side. The study found that the braking and subsequent reversal of the dispersed water droplets significantly affect the initial droplet size Rm. Drops with a diameter less than 0.1-0.2 mm, were unable to overcome the resistance of the counter of high-temperature environment, leading to a reversal flow and drip subsequent entrainment gases. Drops of the best preserved original diameter direction and reached the source of fire. [3]

\section{Conclusion}

Thus, at present and in the near future fire extinguishing based on water mist are the most effective means of fighting the fire, provided their application in accordance with the results of research, namely the necessary spatial differentiation droplet size (relatively large drops should be placed on the perimeter flow finer droplets) considering the characteristics of the equipment.. 


\section{Smart Grids 2015}

Research carried out at the expense of the grant RNF 14-39-00003.

\section{References}

1. Di Wu, Delphine Guillemin, André W. Marshall, Fire Saf. J., 42, 283 (2007)

2. Xiangyang Zhou, Hong-Zeng Yu, Fire Saf. J., 46, 140 (2011)

3. Volkov R.S., Kuznetsov G.V., Strizhak P.A., Thermophysics and Aeromechanics, 21, 255 (2014) 